\title{
Bem-Estar Subjetivo infantil: avaliação por meio do Desenho da Figura Humana
}

\author{
Vanisa Fante Viapiana ${ }^{1}$ \\ Pontifícia Universidade Católica do Rio Grande do Sul - PUCRS, Porto Alegre-RS, Brasil \\ Cláudia de Moraes Bandeira, Claudia Hofheinz Giacomoni \\ Universidade Federal do Rio Grande do Sul - UFRGS , Porto Alegre-RS, Brasil
}

\section{RESUMO}

O objetivo deste estudo foi desenvolver um sistema de avaliação de Bem-Estar Subjetivo (BES) infantil por meio do Desenho da Figura Humana (DFH), bem como apresentar evidências de validade dele. Para tanto, o estudo foi dividido em duas etapas. Na primeira etapa, foram realizados três grupos focais, um com experts em desenvolvimento infantil e os outros dois com crianças entre 8 e 10 anos de idade. Os grupos geraram afirmações sobre características de crianças felizes que, após passarem por juízes treinados, resultaram em 18 itens de avaliação do BES no DFH. Na segunda etapa do estudo, a validade dos itens foi testada. Para tal, participaram 50 crianças com faixa etária entre 8 e 10 anos de idade, que fizeram um desenho da figura humana e responderam escalas de autorrelato de BES. Análises correlacionais mostraram que aspectos do DFH de crianças podem ser considerados indicadores de características positivas do desenvolvimento humano.

Palavras-chave: avaliação psicológica, desenho da figura humana, bem-estar subjetivo.

\section{ABSTRACT - Subjective Well-Being in childhood: Assessment using the Draw-A-Person test}

This study aimed to develop a Children's Subjective Well-Being (SWB) assessment system through the Human Figure Drawing (HFD), and to present validity evidence. The study was divided into two parts. In the first part, three focus groups were held, including one with experts in child development and two others with children between 8 and 10 years old. The groups generated statements about characteristics of happy children, which, after consideration by trained evaluators, resulted in an 18-item assessment of SWB via HFD. In the second part, a validity study was conducted on the items. For this purpose, 50 children between 8 and 10 years of age drew a human figure and answered a self-reported SWB scale. Correlation analyses showed that some aspects of HFD can be considered indicators of positive characteristics of human development.

Keywords: psychological assessment, human figure drawing, subjective well-being.

\section{RESUMEN - Bienestar Subjetivo infantil: evaluación por el Dibujo de la Figura Humana}

El objetivo de este estudio fue desarrollar un sistema de evaluación de Bienestar Subjetivo (BES) infantil a través del Dibujo de la Figura Humana (DFH), y también presentar evidencias de validez del mismo. El estudio se dividió en dos etapas;en la primer etapa fueron realizados tres grupos de enfoque, uno con expertos en desarrollo infantil y los otros dos, con niños entre 8 y 10 años de edad. Los grupos generaron afirmaciones sobre características de niños felices, que después de pasar por jueces entrenados dieron lugar a 18 ítems de evaluación del BES en el DFH. En la segunda etapa fue probada la validez de los ítems. Con este fin, participaron 50 niños con edades comprendidas entre 8 y 10 años,que hicieron un dibujo de la figura humana y respondieron a escalas de autorelato del BES. Análisis correlacionales mostraron que aspectos del DFH de niños pueden ser considerados indicadores de características positivas del desarrollo humano.

Palabras clave: evaluación psicológica; dibujo de la figura humana; bienestar subjetivo.

Cada vez mais a Psicologia tem se interessado pela avaliação e construção de características humanas positivas (Seligman \& Csikzentmihalvi, 2000). Nessa perspectiva, inserem-se os estudos sobre o Bem-Estar Subjetivo (BES). O BES é considerado um construto multidimensional (Segabinazi, Giacomoni, Dias,
Teixeira, \& Moraes, 2010), que se caracteriza pela avaliação cognitiva e emocional que o ser humano faz da própria existência (Diener, Suh, Lucas, \& Smith, 1999). O componente cognitivo do BES refere-se à satisfação de vida, a qual corresponde a uma avaliação de longo prazo (Bradshaw, Keung, Rees, \& Goswami, 2010), 
global ou de domínios específicos (Ben-Zur, 2003) que uma pessoa faz de sua vida. E o componente emocional se caracteriza pela intensidade e frequência com que as pessoas vivenciam afetos positivos e negativos (Lyubomirsky, King, \& Diener, 2005).

A avaliação do BES é tipicamente realizada por meio de escalas de autorrelato (Diener, 1994) unidimensionais ou multidimensionais, únicas ou múltiplas (Pollard \& Lee, 2003). Os estudos direcionados ao BES, na infância, são recentes e numa quantidade inferior aos estudos realizados com adultos (Huebner \& Diener, 2008). No Brasil, foram desenvolvidas diferentes escalas para avaliar os componentes do BES infantil. No que se refere à satisfação de vida infantil, os estudos para construção da Escala Multidimensional de Satisfação de Vida para Crianças (EMSVC), (Giacomoni \& Hutz, 2008) identificaram seis fatores principais para a satisfação de vida de crianças brasileiras: Família, Amigos, Escola, Self, Não violência e Self comparado. Os estudos de validação das Escalas de Afeto Positivo e Negativo para crianças mostram os domínios Self, Família e Amizade como os principais domínios que promovem afeto positivo na infância (Giacomoni \& Hutz, 2006).

As escalas são instrumentos amplamente utilizados na avaliação psicológica, no entanto apresentam algumas limitações, tal como a desejabilidade social. Nessa perspectiva, o Desenho da Figura Humana (DFH) é uma técnica amplamente utilizada para avaliar aspectos cognitivos e emocionais das crianças. O desenho é proposto sob a premissa de que é uma atividade familiar a qualquer criança que, ao desenhar, expressa aspectos de seu funcionamento psicológico e cognitivo (Wechsler, 2012).

O DFH foi, primeiramente, considerado uma medida do desenvolvimento cognitivo infantil, a partir dos estudos de Goodnough, em 1929 (Arden, Trzaskowski, Garfield, \& Plomin, 2014; Nunes, Teixeira, Feil, \& Paniagua, 2012). No entanto, foi verificado que crianças com dificuldades emocionais não conseguiam desenhar uma pessoa conforme sua capacidade intelectual, medida por meio de QI (Nunes et al., 2012). Na busca de critérios de avaliação adequados, foram desenvolvidos diversos sistemas de interpretação do desenho. Alguns sistemas consideram o DFH uma medida projetiva, como os trabalhos de Machover (1949). Já, outros propõem a análise dos indicadores emocionais do DFH por meio de uma análise empírica como os estudos de Koppitz (1984).

$\mathrm{Na}$ literatura da área, não há um consenso sobre os melhores métodos de avaliação do DFH (Arteche \& Bandeira, 2006). Alguns estudos indicam que a diferença dos desenhos infantis se dá por maturidade cognitiva (Arden et al., 2014; Ozer, 2010), e outros consideram o DFH um bom instrumento para avaliar características de personalidade, tais como o estudo de Silva, Paludo, e Costa (2012), que identificou a técnica da figura humana na chuva de Machover (1949) como potencial para avaliar crianças a partir de oito anos, e o estudo de Arteche (2006) que considerou DFH um instrumento de rastreio (screening) clínico, apresentando diferenças significativas entre os desenhos de crianças com demandas clínicas e crianças saudáveis .

Estudos empíricos utilizaram o DFH para avaliar o estado emocional de crianças pré-escolares (Daglioglu, Deniz, \& Kan, 2010), para diferenciar crianças que sofreram violência física e sexual de crianças de um grupo controle (Albornoz, 2011), e para avaliar o bem-estar de crianças hospitalizadas com cardiopatia congênita (Dolidze, Smith, \& Tchanturia, 2013). Em sua maioria, os estudos com o DFH objetivam avaliar ocorrências atípicas no desenvolvimento ou psicopatologias.

Groth-Marnat e Roberts (1998) utilizaram o DFH e o House-Tree-Person (HTP) para avaliar autoestima de universitários. Os pesquisadores definiram nove indicadores do DFH, como presença de detalhes essenciais, proporção adequada da figura, tamanho moderado, qualidade da linha firme, forte aparência, abertura da figura, posição central, potencial de movimento e diferenciação sexual clara. Destes, presença de detalhes essenciais e proporção adequada da figura tiveram correlação positiva para bom nível de autoestima do jovem adulto.

Um estudo realizado por Carboni e Morrow (2011), na Zambia, investigou o bem-estar psicológico de crianças por meio do DFH. Os pesquisadores solicitaram que as crianças desenhassem uma criança que se sentisse bem e uma que se sentisse mal. Nos desenhos que representavam as crianças que se sentiam bem, foram observados detalhes como mochila da escola, sapatos nos pés, roupas limpas e cabelos bem penteados. Por outro lado, nas figuras de crianças que apresentavam mal-estar, percebeu-se a falta de sapatos, cabelos despenteados, roupas sujas e rasgada.

Apesar de o DFH ser uma técnica amplamente utilizada para avaliação psicológica infantil, ainda é raro que o desenho seja utilizado como instrumento de avaliação de aspectos positivos do desenvolvimento humano. Portanto, este estudo teve como objetivo construir um sistema de avaliação de BES infantil por meio do DFH e foi realizado em duas etapas sequenciais. A primeira etapa tratou da elaboração de indicadores de BES e a segunda buscou apresentar evidências preliminares de validade do sistema de avaliação proposto. Essas etapas estão descritas a seguir.

\section{Etapa 1}

A Etapa 1 teve como objetivo construir um sistema de avaliação de BES infantil por meio do DFH. Com o intuito de definir operacionalmente o construto de BES infantil e garantir validade de conteúdo aos indicadores propostos, utilizou-se a técnica dos grupos focais. Os grupos focais têm sido comumente utilizados com o propósito de desenvolver instrumentos psicológicos, pois possibilitam uma análise prévia do que é relevante para o tema e provocam insights de como os itens de um instrumento devem ser apresentados (Baurbour, 2009; 
Gandin, 2002). Realizaram-se três grupos focais de natureza distintas. Um grupo foi composto por experts da área da Psicologia e os demais contaram com a participação de crianças com faixa etária entre 8 e 10 anos de idade.

\section{Método}

\section{Grupos focais}

O grupo focal de experts foi composto por cinco psicólogos e uma estudante de Psicologia. Todos os integrantes possuíam conhecimentos prévios em desenvolvimento infantil, Avaliação Psicológica e Psicologia Positiva.

Apesar da literatura sobre a realização de grupo focal orientar que ele seja dirigido por meio de tópicos (Barbour, 2009; Iervolino \& Pelicioni, 2001), optou-se por perguntas exploratórias, pois estas responderiam de forma mais satisfatória o objetivo de discutir sobre o tema felicidade infantil e sua expressão no DFH. Elaborou-se um roteiro de quatro perguntas 1. Como é uma criança feliz? 2. Como identifica-se que uma criança é feliz? 3. Qual a impressão global que o desenho de uma criança feliz passaria para o avaliador? 4. Quais os itens da figura humana que poderiam ser considerados indicadores de BES?

Além do grupo focal com experts na área de Psicologia considerou-se importante entender a percepção das próprias crianças a respeito do significado do bem-estar subjetivo/felicidade e suas formas de expressões no desenho. Sendo assim, foram realizados dois grupos focais em uma escola pública do Estado do Rio Grande do Sul. Primeiramente, selecionou-se randomicamente, por meio da lista de chamada dos professores, 14 crianças de 8 a 10 anos de idade, 7 meninas e 7 meninos que cursavam do $3^{\circ}$ ao $5^{\circ}$ ano do ensino fundamental. Após a assinatura do Termo de Consentimento Livre e Esclarecido pelos responsáveis, foram formados dois grupos, cada um destes com sete crianças de ambos os sexos. Os grupos contaram com duas psicólogas que atuaram como moderadoras. Durante a reunião do grupo, o termo "bem-estar subjetivo" foi substituído por "felicidade". Apesar das diferenças teóricas entre o construto de BES e de felicidade, a qual é influenciada imediatamente por experiências agradáveis e desagradáveis (Bradshaw et al., 2010), no senso comum, ambos construtos são considerados como sinônimos. Portanto, buscou-se facilitar a discussão.

Solicitou-se que cada criança desenhasse uma figura humana e, então, uma figura humana feliz. Com os desenhos em mãos, as crianças foram convidadas a participar de uma discussão a respeito da felicidade e de como seus desenhos representavam tal construto.

O roteiro de discussão do grupo focal procurou contemplar os objetivos do presente estudo. Para tanto, a discussão foi dividida em três questões. A primeira pergunta exploratória "O que é felicidade?" buscou investigar o conceito de felicidade, a segunda pergunta "Como é uma pessoa feliz?" objetivou definir características de uma pessoa feliz, e, por fim, a terceira pergunta: "O que neste desenho mostra que esta pessoa está feliz?” visou identificar indicadores de felicidade no DFH feito pelas crianças.

As discussões dos três grupos focais foram gravadas e transcritas na íntegra. Posteriormente, os dados foram submetidos a uma análise de conteúdo qualitativa. Seguiu-se o modelo proposto por Bardin (1977), o qual organiza os procedimentos da análise em três fases: 1. pré-análise, 2. exploração do material e 3. tratamento dos resultados, inferência e interpretação. Primeiramente, parafraseou-se o material, excluindo paráfrases menos relevantes e repetidas e, então, agruparam-se as similares. Os dados foram organizados por códigos temáticos, definidos a priori de acordo com as perguntas exploratórias dos grupos focais e geraram subcategorias a posteriori, de acordo com a interpretação das respostas dadas pelos experts e pelas crianças. Além da análise de conteúdo, buscou-se identificar características do desenho da figura humana feliz realizado pelas crianças.

\section{Resultados}

\section{Grupo focal de experts}

A análise de conteúdo possibilitou a construção de diferentes categorias e subcategorias para cada uma das perguntas exploratórias do grupo focal (Tabela 1).

Tabela 1

Categorias Extraídas da Análise de Conteúdo do Grupo Focal de Experts

\begin{tabular}{|c|c|c|c|}
\hline Categoria & Subcategorias & Descrição & Segmentos de fala \\
\hline \multirow{4}{*}{$\begin{array}{l}\text { Características } \\
\text { de uma criança } \\
\text { feliz }\end{array}$} & $\begin{array}{l}\text { Sentimento de } \\
\text { segurança }\end{array}$ & $\begin{array}{l}\text { Crianças que se sentem seguras de } \\
\text { ser amadas em diferentes ambientes. }\end{array}$ & $\begin{array}{l}\text { "sente o amor dos pais", "pode errar } \\
\text { (...) não vai deixar de ser amada". }\end{array}$ \\
\hline & Curiosidade & $\begin{array}{l}\text { Exploração do ambiente de forma } \\
\text { curiosa. }\end{array}$ & $\begin{array}{l}\text { "quer conhecer o que a cerca", } \\
\text { "busca aprender". }\end{array}$ \\
\hline & Brincadeiras & $\begin{array}{l}\text { Refere-se ao lazer e ao uso da } \\
\text { criatividade. }\end{array}$ & $\begin{array}{l}\text { "tempo suficiente para brincar", } \\
\text { "cria suas brincadeiras", "fantasia } \\
\text { situações felizes". }\end{array}$ \\
\hline & $\begin{array}{l}\text { Características de } \\
\text { extroversão }\end{array}$ & $\begin{array}{l}\text { Refere-se às habilidades de } \\
\text { socialização. }\end{array}$ & $\begin{array}{l}\text { "criança falante", "expressa o que } \\
\text { sente e pensa". }\end{array}$ \\
\hline
\end{tabular}


Tabela 1 (continuação)

Categorias Extraídas da Análise de Conteúdo do Grupo Focal de Experts

\begin{tabular}{|c|c|c|c|}
\hline Categoria & Subcategorias & Descrição & Segmentos de fala \\
\hline \multirow{3}{*}{$\begin{array}{l}\text { Identificação } \\
\text { da felicidade } \\
\text { infantil }\end{array}$} & Aparência & $\begin{array}{l}\text { Percepção de que a criança recebe } \\
\text { cuidados básicos. }\end{array}$ & $\begin{array}{l}\text { "tu vê e percebe que alguém cuida } \\
\text { dela", } \\
\text { "está com a roupa limpa". }\end{array}$ \\
\hline & Comportamento & $\begin{array}{l}\text { Comportamentos que possibilitam a } \\
\text { boa relação com o ambiente. }\end{array}$ & $\begin{array}{l}\text { "busca interação com os pares", } \\
\text { "consegue controlar agressividade". }\end{array}$ \\
\hline & $\begin{array}{l}\text { Expressão de } \\
\text { sentimento }\end{array}$ & $\begin{array}{l}\text { Crianças que expressam sentimentos } \\
\text { positivos. }\end{array}$ & $\begin{array}{l}\text { "demonstra afeto com as pessoas e } \\
\text { animais", } \\
\text { "expressa alegria" }\end{array}$ \\
\hline \multirow{6}{*}{$\begin{array}{l}\text { Indicadores de } \\
\text { felicidade/BES } \\
\text { no DFH }\end{array}$} & $\begin{array}{l}\text { Representação de } \\
\text { afetos positivos }\end{array}$ & $\begin{array}{l}\text { Desenho expressa vivências e } \\
\text { sentimentos positivos da vida } \\
\text { humana. }\end{array}$ & $\begin{array}{l}\text { "a pessoa parece feliz, está sorrindo", } \\
\text { "desenho de coração", "cores } \\
\text { alegres", "desenham contando algo } \\
\text { legal que aconteceu". }\end{array}$ \\
\hline & $\begin{array}{l}\text { Aparência } \\
\text { agradável }\end{array}$ & $\begin{array}{l}\text { Desenho com boa aparência, com } \\
\text { aspecto limpo. }\end{array}$ & $\begin{array}{l}\text { "ela capricha no desenho, do jeitinho } \\
\text { dela", "um desenho sem muitas } \\
\text { rasuras". }\end{array}$ \\
\hline & Integração & $\begin{array}{l}\text { Integração das linhas e ausência de } \\
\text { linhas cruzadas. }\end{array}$ & $\begin{array}{l}\text { "desenha o quadrado, sem cruzar } \\
\text { uma linha na outra", "as linhas, } \\
\text { mesmo tortas são feitas com } \\
\text { cuidado". }\end{array}$ \\
\hline & Normalidade & $\begin{array}{l}\text { Desenho com características reais } \\
\text { do corpo humano (dependendo da } \\
\text { idade). }\end{array}$ & $\begin{array}{l}\text { "A criança desenha a cabeça, o } \\
\text { tronco, os membros". }\end{array}$ \\
\hline & Linha & Qualidade da linha. & $\begin{array}{l}\text { "Não desenha tão forte, que remeta a } \\
\text { muita agressividade" } \\
\text { "energia pra não desenhar com } \\
\text { aquela linha tão fraquinha, que mal } \\
\text { se enxerga". }\end{array}$ \\
\hline & $\begin{array}{l}\text { Presença de } \\
\text { detalhes }\end{array}$ & $\begin{array}{l}\text { Quantidade de adereços e enfeites } \\
\text { no desenho. }\end{array}$ & $\begin{array}{l}\text { "se ela enfeita, percebe investimento } \\
\text { da criança ao desenhar", "o desenho } \\
\text { tem flor, coração ou algo que ele } \\
\text { gosta". }\end{array}$ \\
\hline
\end{tabular}

\section{Grupos focais realizados com crianças}

A análise de conteúdo dos dados decorrentes dos grupos focais infantis resultou em categorias e subcategorias que são descritas na Tabela 2.

Além da análise de conteúdo dos dados dos grupos focais, foram analisados os desenhos da figura humana e da figura humana feliz, feitos pelas crianças. Dessa etapa, resultaram 28 desenhos, 14 da figura humana e 14 da figura humana feliz. Buscou-se identificar diferenças existentes entre os dois desenhos realizados por cada criança.
Observou-se que elas utilizaram estratégias semelhantes para demonstrar que a segunda figura representava uma pessoa feliz, tais como: maior número de presença de objetos inanimados, como sol e nuvens sorrindo, maior presença de elementos da natureza e símbolos afetivos, como o coração. Das quatorze crianças participantes dos grupos focais, oito desenharam a primeira figura com os braços estendidos junto ao corpo, e a segunda, que caracterizava uma pessoa feliz, com os braços abertos ou erguidos.

Tabela 2

Categorias Extraídas da Análise de Conteúdo dos Grupos Focais de Crianças

\begin{tabular}{llll}
\hline Categorias & Subcategorias & Descrição & Segmentos de fala \\
\hline $\begin{array}{l}\text { Características } \\
\text { de uma pessoa }\end{array}$ & Amizade & Convivência com os amigos, ter & "ter bastante amigos", \\
feliz & & amigos, fazer novos amigos. & "fazer novos amigos", \\
& & "ir na casa dos amigos". \\
\hline
\end{tabular}


Tabela 2 (continuação)

Categorias Extraídas da Análise de Conteúdo dos Grupos Focais de Crianças

\begin{tabular}{|c|c|c|c|}
\hline Categorias & Subcategorias & Descrição & Segmentos de fala \\
\hline \multirow{7}{*}{$\begin{array}{l}\text { Características } \\
\text { de uma pessoa } \\
\text { feliz }\end{array}$} & Família & Afeto familiar. & $\begin{array}{l}\text { "ter carinho da família", } \\
\text { "ter amor pelos pais", } \\
\text { "ter amor dos pais". }\end{array}$ \\
\hline & Afetos positivos & Vivência de afetos positivos. & $\begin{array}{l}\text { "estar de bem com a vida", "ter } \\
\text { amor", "ter paz". }\end{array}$ \\
\hline & $\begin{array}{l}\text { Ausência de afetos } \\
\text { negativos }\end{array}$ & $\begin{array}{l}\text { Ausência de vivência de afetos } \\
\text { negativos. }\end{array}$ & $\begin{array}{l}\text { "não pensar maldades", } \\
\text { "não ter preocupações". }\end{array}$ \\
\hline & Altruísmo & $\begin{array}{l}\text { Comportamentos em favor do outro } \\
\text { e socialmente esperados. }\end{array}$ & $\begin{array}{l}\text { “ajudar quem você pode”, } \\
\text { "tentar ajudar". }\end{array}$ \\
\hline & Ausência de brigas & Evitar brigas com amigos e família. & $\begin{array}{l}\text { "Ninguém brigar com você", "não } \\
\text { brigar". }\end{array}$ \\
\hline & Escola & Aprendizagem escolar. & $\begin{array}{l}\text { "quem vai na escola”, } \\
\text { "quem aprende". }\end{array}$ \\
\hline & Lazer & $\begin{array}{l}\text { Situações de brincadeiras, passeios e } \\
\text { viagens. }\end{array}$ & $\begin{array}{l}\text { "ir no parque", } \\
\text { "jogar videogame", } \\
\text { "andar de bicicleta", }\end{array}$ \\
\hline \multirow{4}{*}{$\begin{array}{l}\text { Indicadores de } \\
\text { felicidade no } \\
\text { DFH }\end{array}$} & $\begin{array}{l}\text { Elementos da } \\
\text { natureza }\end{array}$ & $\begin{array}{l}\text { Contato com a natureza, a } \\
\text { possibilidade de cuidar do meio } \\
\text { ambiente, bem como de usufruir dos } \\
\text { benefícios oferecidos por ele. }\end{array}$ & $\begin{array}{l}\text { "está feliz porque está cuidando da } \\
\text { florzinha", "feliz pegando uma fruta } \\
\text { na árvore", "está tranquilo no meio } \\
\text { das flor". }\end{array}$ \\
\hline & Expressão facial & $\begin{array}{l}\text { Pessoa que expressa felicidade por } \\
\text { meio das expressões faciais. }\end{array}$ & $\begin{array}{l}\text { "dá risada", "sorri todos os dias", “os } \\
\text { olhos, eles brilham de felicidade". }\end{array}$ \\
\hline & Expressão corporal & $\begin{array}{l}\text { Expressão de felicidade } \\
\text { por meio dos braços erguidos, } \\
\text { indicando comemoração }\end{array}$ & $\begin{array}{l}\text { "está feliz com os braços } \\
\text { levantados", "fazendo uhul". }\end{array}$ \\
\hline & $\begin{array}{l}\text { Objetos de } \\
\text { valor financeiro }\end{array}$ & $\begin{array}{l}\text { Presença de } \\
\text { bens materiais. }\end{array}$ & $\begin{array}{l}\text { "está feliz porque está em um carro", } \\
\text { "encontrou o tesouro". }\end{array}$ \\
\hline
\end{tabular}

A partir dos dados obtidos por meio das observações dos desenhos e das discussões dos grupos focais, desenvolveu-se 18 indicadores que compuseram o sistema de avaliação BES/DFH (Tabela 3).

Tabela 3

Itens de Avaliação do BES através do DFH

1. Linhas firmes, bem controladas, sem nenhuma ondulação (mais da metade da figura).

2. União das linhas: os pontos de união das linhas devem encontrar-se sem tendência a se cruzar, sem espaço entre as extremidades, considerando o desenho como um todo.

3. Integração da figura: a cabeça unida ao pescoço ou topo do tronco, dois braços unidos ao limite superior do tronco, duas pernas unidas ao limite inferior.

4. Proporção adequada: existe adequação dos membros (inferiores e superiores) em relação ao tamanho do corpo.

5. Simetria adequada: ambos os braços e pernas têm a mesma forma.

6. Limpeza: figura com bom aspecto, falta de rasuras, borrões e linhas apagadas.

7. Tamanho médio da figura: o tamanho total do desenho da figura humana em sua altura deve estar entre 6 $\mathrm{cm}$ e $22 \mathrm{~cm}$.

8. Figura central: o desenho ocupa os 4 quadrantes da folha. 
Tabela 3 (continuação)

Itens de Avaliação do BES através do DFH

9. Pano de fundo: presença de elementos da natureza, animais, automóveis, construções, sol, lua, nuvens, arco-íris.

10. Símbolos afetivos: desenho de corações, sorrisos, escritas ou outros detalhes que representam afeto (não inclui a face da figura humana sorrindo).

11. Penteado: qualquer tentativa de representar um corte ou penteado dos cabelos.

12. Boca expressando sorriso (por exemplo: boca em forma de meia lua, com uma ou duas linhas)

13. Braços não estendidos: ao menos um braço afastado do tronco, em um ângulo igual ou maior que $45^{\circ}$.

14. Roupa: pontua-se se há a presença de alguma peça de roupa no desenho da figura humana: calças, bermudas, saias, vestido, etc.

15. Sapato: qualquer tentativa de representar um sapato.

16. Detalhes: Pontua-se se houver algum tipo de detalhe no desenho da figura humana (qualquer detalhe nas vestimentas, como botões e estampas, acessórios, joias, óculos, cadarços, artigos esportivos, brinquedos exclui-se símbolos agressivos).

17. Objetos: quando há a presença de um ou mais objetos junto à figura ou sendo portados por ela (exclui-se símbolos agressivos).

18. Objetos inanimados: características humanas em desenhos não humanos (ex.: sol com rosto, balões com rosto, etc.)

\section{Etapa 2}

A segunda etapa deste estudo testou empiricamente o sistema de avaliação BES/DFH por meio da análise de desenhos de crianças escolares. O objetivo deste estudo foi verificar a validade de construto e concorrente dele.

\section{Método}

\section{Participantes}

Esta pesquisa contou com 50 desenhos realizados por estudantes de escolas particulares da região metropolitana de Porto Alegre/RS. Os desenhos foram selecionados de um banco de dados de um projeto de pesquisa sobre desenvolvimento positivo do Laboratório de Mensuração da Universidade Federal do Rio Grande do Sul (UFRGS). A amostra foi pareada quanto ao sexo (25 meninos) e composta por estudantes de $2^{\circ}$ a $5^{\circ}$ ano do ensino fundamental, com faixa etária entre 8 e 10 anos de idade $(M=8,86 ; D P=0,80)$. Todos faziam parte de uma população não clínica e apresentavam altos níveis de BES, conforme a Escala Multidimensional de Satisfação de Vida (EMSVC) - versão reduzida (Zanon, Bandeira, \& Giacomoni, no prelo) $(M=4,52, D P=0,28)$, a Escala de Afetos Positivos e Negativos (Giacomoni \& Hutz, 2006) $(M=4,30 ; D P=0,45 ; M=1,77 ; D P=0,82$, respectivamente).

\section{Instrumentos}

Escala de Afeto Positivo e Negativo (Giacomoni \& Hutz, 2006). Composta por 30 itens, sendo 15 itens da subescala de afeto positivo e 15 itens da subscala de afeto negativo. É utilizada uma escala de respostas do tipo Likert de cinco pontos, que variam de um (nem um pouco) a cinco (muitíssimo). O coeficiente alfa da escala total, nos estudos de construção foi de 0,90 (Giacomoni \& Hutz, 2006). Para a subescala de Afeto Positivo, esse índice foi de 0,88 e, para a subescala de Afeto Negativo, foi de 0,84 , sendo considerados consistentemente satisfatórios.

Escala Multidimensional de Satisfação de Vida (EMSVC) - versão reduzida (Zanon et al., no prelo). Distribuída em quatro fatores: Self comparado, Família, Escola e Amizade. A avaliação é realizada por meio de uma escala Likert que varia de um (nem um pouco) a cinco (muitíssimo) pontos. O alfa obtido para a escala total foi considerado elevado, acima de 0.90 .

Escala Global de Avaliação do Desenho (Segabinazi, 2010). Pontua o DFH por meio de uma escala Likert de cinco pontos, em três dimensões: normalidade, qualidade artística e diferenciação sexual.

Compêndio de Indicadores Emocionais desenvolvido por Albornoz (2011) com base no estudo de Arteche (2006) sobre itens de avaliação emocional do DFH. O compêndio reuni 143 itens dicotômicos de presença e ausência.

\section{Procedimentos}

Os 50 desenhos foram avaliados por três juízes independentes, psicólogos previamente treinados, por meio do sistema de avaliação BES/DFH construído na etapa 1 deste estudo, do Compêndio de Indicadores Emocionais de Albornoz (2011) e da Escala de Avaliação Global do Desenho de Segabinazi 
(2010). Posteriormente, cada desenho foi pontuado de acordo com a mensuração da moda extraída das análises dos juízes.

Examinou-se os padrões correlacionais entre os sistemas de avaliação de BES/DFH e a Escala Global de Avaliação do Desenho, a fim de averiguar validade concorrente dos itens. E, então, realizou-se estudos de correlação de Pearson entre o sistema de avaliação do BES/DFH, as Escalas de Afeto Positivo e Negativo e a EMSVC. Assim como do Compêndio de Indicadores Emocionais e das Escalas de Afetos Positivo e Negativo e a EMSVC.

\section{Resultados}

A avaliação dos desenhos realizada por três juízes passaram por uma análise de frequência e obtiveram níveis satisfatórios de concordância. $\mathrm{O}$ sistema de avaliação BES/DFH obteve $91 \%$ de concordância entre juízes. Já o Compêndio de Indicadores Emocionais apresentou um nível de concordância de 96\%, e a Escala de Avaliação Global do DFH de 71\%.

Os itens do sistema de avaliação de BES/DFH foram correlacionados com as três medidas da Escala de Avaliação Global do Desenho (Tabela 4).

Tabela 4

Correlações entre o Sistema de Avaliação de BES/DFH e a Escala Global de Avaliação do Desenho

\begin{tabular}{|c|c|c|c|}
\hline \multirow[b]{2}{*}{$\mathrm{BES} / \mathrm{DFH}$} & \multicolumn{3}{|c|}{ Escala de Avaliação Global do DFH } \\
\hline & $\begin{array}{c}\text { Qualidade } \\
\text { artística }\end{array}$ & Normalidade & $\begin{array}{c}\text { Diferenciação } \\
\text { sexual }\end{array}$ \\
\hline Linhas firmes & $0,60^{* *}$ & $0,37^{*}$ & 0,22 \\
\hline União das linhas & $0,28^{*}$ & $0,28^{*}$ & $-0,06$ \\
\hline Integração da figura & $\mathrm{B}$ & B & $\mathrm{B}$ \\
\hline Proporção adequada & $0,34^{*}$ & $0,39^{*}$ & 0,22 \\
\hline Simetria adequada & 0,25 & 0,23 & 0,11 \\
\hline Limpeza & $0,45^{* *}$ & 0,28 & 0,18 \\
\hline Tamanho médio da figura & 0,21 & 0,20 & 0,10 \\
\hline Figura central & $-0,02$ & $-0,02$ & 0,14 \\
\hline Pano de fundo & 0,01 & $-0,16$ & 0,20 \\
\hline Símbolos afetivos & $-0,20$ & $-0,08$ & 0,12 \\
\hline Penteado & 0,23 & 0,23 & $0,55^{* *}$ \\
\hline Boca expressando sorrindo & $-0,32$ & 0,45 & 0,01 \\
\hline Braços não estendidos & 0,16 & 0,13 & 0,10 \\
\hline Roupa & 0,21 & $0,31^{*}$ & $?$ \\
\hline Sapato & $0,45^{* *}$ & $0,31^{*}$ & $0,35^{*}$ \\
\hline Detalhes & 0,20 & 0,13 & 0,25 \\
\hline Detalhes de gênero da figura & $0,40^{* *}$ & $0,35^{*}$ & $0,66^{* *}$ \\
\hline Objetos inanimados & $-0,02$ & $-0,03$ & $-0,01$ \\
\hline
\end{tabular}

Nota. O indicador "Integração da figura" não pôde ser correlacionado por ausência de variância

Em seguida, analisou-se os padrões correlacionais das escalas de autorrelato de BES infantil em relação ao sistema de avaliação BES/DFH (Tabela 5) e ao
Compêndio de Indicadores Emocionais (Tabela 6), devido à extensão do número de itens desse instrumento são apresentados os resultados com correlação acima de 0,3.

Tabela 5

Correlações entre o Sistema de Avaliação de BES/DFH e as Escalas de BES para Crianças

\begin{tabular}{lccc}
\multicolumn{1}{c}{ BES/DFH } & $\begin{array}{c}M \\
\text { afeto positivo }\end{array}$ & $\begin{array}{c}\text { M } \\
\text { afeto negativo }\end{array}$ & $\begin{array}{c}\text { satisfação de vida } \\
\text { Linhas firmes }\end{array}$ \\
União das linhas & 0,14 & 0,12 & 0,10 \\
Integração da figura & $-0,27$ & 0,09 & 0,06 \\
Proporção adequada & $\mathbf{0 , 3 0 *}$ & 0,09 & $\mathbf{0 , 3 9 * *}$ \\
Simetria adequada & 0,03 & 0,15 & $-0,09$ \\
\hline
\end{tabular}


Tabela 5 (continuação)

Correlações entre o Sistema de Avaliação de BES/DFH e as Escalas de BES para Crianças

\begin{tabular}{lccc}
\hline \multicolumn{1}{c}{ BES/DFH } & $\begin{array}{c}\text { M } \\
\text { afeto positivo }\end{array}$ & $\begin{array}{c}\text { M } \\
\text { afeto negativo }\end{array}$ & $\begin{array}{c}\text { M } \\
\text { satisfação de vida }\end{array}$ \\
\hline Limpeza & 0,11 & 0,25 & 0,04 \\
Tamanho médio da figura & 0,03 & 0,08 & 0,11 \\
Pano de fundo & 0,09 & $-0,16$ & 0,16 \\
Figura central & 0,27 & $-0,13$ & $\mathbf{0 , 3 3 ^ { * }}$ \\
Símbolos afetivos & 0,20 & 0,20 & 0,17 \\
Penteado & 0,19 & $-0,22$ & 0,11 \\
Boca expressando sorriso & $-0,30^{*}$ & 0,13 & 0,01 \\
Braços não estendidos & 0,20 & 0,16 & 0,24 \\
Roupa & $-0,03$ & 0,12 & 0,05 \\
Sapato & 0,11 & 0,11 & 0,11 \\
Detalhes & $-0,33^{*}$ & $-0,09$ & $-0,11$ \\
Detalhes de gênero da figura & 0,15 & $-0,15$ & $\mathbf{0 , 3 8 ^ { * * }}$ \\
Objetos inanimados & 0,17 & 0,20 & 0,05 \\
Objetos & 0,20 & $-0,07$ & 0,02 \\
\hline
\end{tabular}

Nota. ${ }^{*} p \leq 0,05^{* *} p \leq 0,01$

Legenda. Domínios da EMSVC: Self comparado, Família, Amizade e Escola

Tabela 6

Correlações entre o Compêndio de Indicadores Emocionais e as Escalas de BES para Crianças

\begin{tabular}{|c|c|c|c|}
\hline Compêndio de indicadores emocionais & $\begin{array}{c}M \\
\text { afeto positivo }\end{array}$ & $\begin{array}{c}M \\
\text { afeto negativo }\end{array}$ & $\begin{array}{c}M \\
\text { satisfação de vida }\end{array}$ \\
\hline Desenho da linha de base & & $-0,31^{*}$ & \\
\hline Omissão do cabelo & $-0,33^{* *}$ & & \\
\hline Ênfase no nariz & $-0,37^{* *}$ & & \\
\hline Ênfase no chapéu & & $0,49 * *$ & \\
\hline Desproporção braços e pernas & $-0,32^{*}$ & & \\
\hline Objetos & & & $-0,40^{*}$ \\
\hline Figura grande & & & $-0,36^{*}$ \\
\hline Nariz pequeno & & & $0,43^{* *}$ \\
\hline Pernas com linhas esboçadas & & & $-0,38^{* *}$ \\
\hline Pano de fundo & & & $0,30^{*}$ \\
\hline
\end{tabular}

Nota. ${ }^{*} p \leq 0,05{ }^{* *} p \leq 0,01$

Legenda. Domínios da EMSVC: Self comparado, Família, Amizade e Escola

\section{Discussão}

Este estudo objetivou a construção de um sistema de avaliação de BES infantil por meio do DFH. Para isso, foi realizado em duas etapas sequenciais. A primeira visou criar os indicadores para o sistema de avaliação proposto e a segunda testou evidências de validade dos itens criados.

A construção de itens baseou-se nas discussões dos grupos focais realizados com crianças e também com experts da área da Psicologia. As categorias e subcategorias extraídas a partir da análise de conteúdo dos dados corroboram os estudos sobre o BES infantil que propõem um modelo multidimensional, por meio da inclusão de dimensões emocionais e cognitivas.
As subcategorias dos grupos focais com crianças: "expressão facial”, "expressão corporal" e "objetos de valor", tornaram-se os itens: "face expressando emoção positiva", "braços não estendidos" e "objetos". A subcategoria referente a "elementos da natureza" foi englobada no item "pano de fundo". Essa subcategoria está de acordo com o estudo de Arteche (2006), que encontrou uma alta ocorrência de indicadores, como "nuvens" e "pano de fundo", que se refere à presença de árvores, flores, etc. em uma amostra de crianças não clínicas.

Segundo as análises resultantes do grupo focal de experts, alguns aspectos são esperados no desenho de uma criança que se sente bem e feliz. Entre eles, encontraram-se aspectos de avaliação global do desenho como "aparência agradável", que resultou nos 
indicadores: "limpeza" e "tamanho da figura média". Os experts referiram também indicadores que são tradicionalmente avaliados na prática psicológica, como a "qualidade" e "interação" da linha e a "normalidade" da figura humana. A linha fina/fraca ou grossa/pesada é analisada, respectivamente, como insegurança ou agressividade (Di Leo, 1987). No estudo de Arteche (2006), a linha pesada foi considerada um item importante para a predição de dificuldades emocionais, especialmente para meninos de 9 a 12 anos. A falta de integralidade, por sua vez, remete aos profissionais pensar em desordens (Di Leo, 1987). Já a normalidade costuma avaliar a proporcionalidade do desenho e a presença de detalhes bizarros (Bandeira \& Hutz, 1995). Portanto, as categorias e subcategorias mostram-se consistentes para fundamentar a escolha de indicadores de BES infantil no desenho, garantindo a validade de conteúdo dos itens que compuseram o sistema de avaliação de BES/DFH.

Posterior à criação do sistema de avaliação de BES/ DFH, testou-se a validade de construto dele. A alta concordância (91\%) entre os três juízes que analisaram os desenhos indica que os itens foram bem formulados e expõem com clareza o que pretendem avaliar. A clareza do item é fundamental para assegurar validade e fidedignidade a um instrumento, especialmente em avaliações como do desenho que pode envolver uma série de questões subjetivas do avaliador.

Os padrões correlacionais entre o sistema de avaliação de BES/DFH e as medidas de qualidade artística, normalidade e diferenciação sexual propostas por Segabinazzi (2010) mostraram-se congruentes. A qualidade da linha traçada, a integração e proporção da figura foram positivamente associadas às medidas de qualidade artística e normalidade. Também conforme o esperado, a "presença de roupas" foi correlacionada positivamente com a medida de normalidade, bem como os itens "sapato", "penteado" e "detalhes que identificam o gênero da figura" com a medida de diferenciação sexual. Tais correlações evidenciam validade concorrente aos itens propostos pelo sistema de avaliação BES/DFH.

Por outro lado, as análises correlacionais entre os itens do sistema de avaliação BES/DFH e das escalas de autorrelato de BES infantil possibilitam evidenciar validade de construto aos itens. Observa-se que nenhum dos itens do sistema de avaliação BES/DFH apresentaram um nível de correlação significativo em relação à Escala de Afeto Negativo. Apesar de o BES ser um construto que envolve o equilíbrio entre a vivência de afetos positivos e negativos, é preciso haver a predominância de afetos positivos para se considerar que uma pessoa se sente bem. Sendo assim, espera-se que uma criança que se sente bem e feliz na maior parte do tempo, expresse esse tipo de afeto em seu desenho.

Os itens "integração da figura", "figura central" e "detalhes que identificam o gênero" mostraram-se associados com as dimensões do BES. Portanto, sugere-se que tais itens sejam considerados indicadores de desenvolvimento positivo infantil. No entanto, ao contrário do que foi esperado pelas pesquisadoras, os itens "boca expressando sorriso" e "detalhes" obtiveram correlações negativas com afeto positivo. Sugere-se que esses itens sejam reanalisados em estudos posteriores, pois, por mais que não tenham apresentado evidências empíricas, teoricamente são fortemente associados à expressão de fatores positivos.

A partir dos padrões correlacionais entre o Compêndio de Indicadores Emocionais (Albornoz, 2011) e as escalas de autorrelato de BES infantil, sugere-se novos itens para serem inclusos no sistema de avaliação de BES/DFH. É possível inferir que "nariz pequeno" é um indicador de BES no DFH, a partir da correlação positiva $(p<0,43)$ com a média de satisfação de vida e da correlação negativa $(p<-0,37)$ do item "ênfase no nariz" com afetos positivos.

Os resultados evidenciaram que os itens de avaliação de BES por meio do desenho melhor se correlacionam com a Escala de Satisfação de Vida do que de Afetos Positivos e Negativos. O que pode ser explicado pela estreita relação do desenho com as habilidades cognitivas das crianças. Considera-se a satisfação de vida como um julgamento cognitivo sobre a própria existência, assim como o desenho pode ser entendido como uma forma de expressão da criança, de como ela julga a si e ao mundo (Wechsler, 2012).

O DFH mostrou-se um instrumento potencial para avaliação de características positivas do desenvolvimento humano. No entanto, é um estudo preliminar que visou criar itens e testar sua validade na forma de um sistema de avaliação, que carece de mais investimento no seu escopo de validade, bem como fidedignidade e normatização. Algumas limitações devem ser consideradas no presente estudo, o qual não avaliou nível cognitivo de cada criança e não diferenciou meninas e meninos. Com o objetivo de controlar variáveis socioeconômicas, optou-se por avaliar desenhos de crianças de escola privada. Porém, se faz necessário que novos estudos sejam conduzidos com crianças de diferentes classes socioeconômicas. Considerando que este estudo tem caráter preliminar e servirá de base para estudos futuros, sugere-se ainda que sejam conduzidos estudos de validade que avalie o desenho de crianças com altos e baixos níveis de BES.

A Psicologia vem se dedicando ao estudo do desenvolvimento positivo desde a infância, no entanto, mais estudos são necessários para o desenvolvimento de técnicas de avaliação de características positivas. O desenho da figura humana assume um importante papel na avaliação psicológica de crianças, o que confirma a necessidade de continuidade nos estudos de aprimoramento de um sistema de avaliação de BES infantil no DFH. 


\section{Referências}

Albornoz, A. C. (2011). Desenho da figura humana: indicadores de abandono, abuso sexual e abuso físico em crianças (Tese de doutorado não publicada). Universidade Federal do Rio Grande do Sul, Porto Alegre RS.

Arden, R. Trzaskowski M., Garfield V., \& Plomin, R. (2014). Genes influence young children's human figure drawings and their association with intelligence a decade later. Psychological Science, 5(10), 1-8. doi:10.1177/0956797614540686

Arteche, A. X. (2006). Indicadores emocionais do desenho da figura humana: construção e validação de uma escala infantil (Tese de doutorado não publicada). Universidade Federal do Rio Grande do Sul, Porto Alegre RS.

Arteche, A., \& Bandeira, D. R. (2006). O desenho da figura humana: revisando mais de um século de controvérsias. Revista Iberoamericana de Diagnóstico y Evaluación Psicológica, 2(22), 133-156. Recuperado de http://www.researchgate.net/publication/240623450_O_desenho_ da_figura_humana_Revisando_mais_de_um_sculo_de_controvrsias_Draw-a-Person_a_century_of_controversies.

Bandeira, D. R., \& Hutz, C, S. (1995). Avaliação psicológica com o desenho da figura humana: técnica ou intuição? Temas em Psicologia, 3(3), 35-41. Recuperado de http://pepsic.bvsalud.org/scielo.php?pid=S1413389X1995000300005\&script=sci_arttext.

Barbour, R. (2009). Grupos Focais. Porto Alegre: Artmed.

Bardin, L. (1977). Análise de Conteúdo. Lisboa: Edições 70.

Bradshaw J., Keung, A., Rees, G., \& Goswami, H. (2010). Children and youth services review children's subjective well-being: International comparative perspectives. Children and Youth Services Review, 33(4), 548-556. doi: 10.1016/j.childyouth.2010.05.010

Ben-Zur, H. (2003). Happy adolescents: The link between subjective well-being, internal resources, and parental factors. Journal of Youth and Adolescence, 32(2), 67-79. doi: 10.1023/A:1021864432505

Carboni, I., \& Morrow, N. (2011). Finding the right balance between standardization and flexibility: A compendium of indicators for measuring child well-being. Child Indicators Research, 4(4), 597-618. doi: 10.1007/s12187-011-9109-3

Daglioglu, H. E., Deniz, U., \& Kan, A. (2010). A study on the emotional indicators in 5-6 year-old girls and boys human figure drawings. Procedia Social and Behavioral Sciences, 2(2), 1503-1510. doi: 10.1016/j.sbspro.2010.03.226

Di Leo, J. H. (1987). A interpretação do desenho infantil. Porto Alegre: Artes Médicas.

Diener, E. (1994). Assessing subjective well-being: Progress and opportunities. Social Indicators Research, 31(2), 103-157. doi: 10.1007/ BF01207052

Diener, E., Suh, E. M., Lucas, R. E., \& Smith, H. L. (1999). Subjective well-being: Three decades of progress. Psychological Bulletin, 2, 276-302. Recuperado de http://ssrn.com/abstract=2199216

Dolidze, K., Smith, E. L., \& Tchanturia, K. (2013). A clinical tool for evaluating emotional well-being: Self-drawings of hospitalized children. Journal of Pediatric Nursing. 28(5) 470-478. doi: 10.1016/j.pedn.2013.02.026

Gandin, S. M. G. (2002). Grupos focais como técnica de investigação qualitativa: desafios metodológicos. São Paulo: Paidéia.

Giacomoni, C. H., \& Hutz, C. S. (2006). Escala de afeto positivo e negativo para crianças: Estudos de construção e validação. Revista Semestral da Associação Brasileira de Psicologia Escolar e Educacional, 10(2), 235-245. doi: 10.1590/S1413-85572006000200007.

Giacomoni, C. H., \& Hutz, C. S. (2008). Escala multidimensional de satisfação de vida para crianças: estudos de construção e validação. Estudos de Psicologia, 25(1), 23-35. doi: 10.1590/S0103-166X2008000100003

Groth-Marnat, \& G. Roberts, L. (1998). Human figure drawing and house tree person drawing as indicators of self-esteem: A quantitative approach. Journal of Clinical Psychology, 54(2), 219-222. doi: 10.1002/(SICI)1097-4679(199802)54:2<219::AIDJCLP11>3.0.CO,2-M

Huebner, E. S., \& Diener, C. (2008). Research on life satisfaction of children and youth. Em M. Eid \& R. J. Larsen. (Eds.), The science of Subjective Well-Being (pp. 376-392). New York, N.Y.: The Guilford Press.

Iervolino, S., A., \& Pelicioni, M., C., F. (2001). A utilização do grupo focal como metodologia qualitativa na promoção da saúde. Revista da Escola de Enfermagem USP, 35(2),115-21. doi: 10.1590/S0080-62342001000200004

Koppitz, E. M. (1984). El dibujo de la figura humana em los niños. Buenos Aires: Guadalupe.

Lyubomirsky, S., King, L., \& Diener, E. (2005). The benefits of frequent positive affect: Does happiness lead to success? Psychological Bolletin, 131(6), 803-855. doi: 10.1037/0033-2909.131.6.803

Machover, K. (1949). Proyección de la personalidad en el dibujo de la figura humana: um método de investigacion de la personalidad. Habana: Cultural.

Nunes, M. L. T, Teixeira, R. P., Feil, C. F., \& Paniagua, R. M. (2012). O desenho da figura humana: uma perspectiva histórica. Em S. M. Wechsler \& T. C. Nakano (Eds.). O desenho infantil - forma de expressão cognitiva, criativa e emocional. (pp. 15-32). São Paulo: Casa do Psicólogo.

Ozer, S. (2010). A comparison of clinical and nonclinical groups of children on the Bender-Gestalt and Draw a Person Tests. Procedia Social and Behavioral Sciences, 5, 449-454. doi:10.1016/j.sbspro.2010.07.121

Pollard, E. L., \& Lee, P.D. (2003). Child well-being: A systematic review of the literature. Social Indicators Research, 61(1), 59-78. doi: 10.1023/A:1021284215801

Segabnazi, J. D., Giacomoni, C. H., Dias, A. C. G., Teixeira, M. A. P., \& Moraes, D. A. (2010). Desenvolvimento e validação preliminar de uma escala multidimensional de satisfação de vida para adolescentes. Psicologia: Teoria e Pesquisa, 26(4), 653-659. doi:10.1590/S010237722010000400009

Segabinazi, J. D. (2010). Desenho da figura humana: evidências de validade de escalas globais de avaliação (Dissertação de mestrado não publicada). Universidade Federal do Rio Grande do Sul, Porto Alegre RS.

Seligman, M. E. P., \& Csikszentmihalyi, M. (2000). Positive psychology: An introduction. American Psychologist, 55(1), 5-14. doi: 10.1037/0003066X.55.1.5.

Silva, R. B. F., Paludo, E., \& Costa, V. S. (2012). O desenho da figura humana na chuva: resgate de uma técnica. Em S. M. Wechsler \& T. C. Nakano (Ed.). O desenho infantil: forma de expressão cognitiva, criativa e emocional. (pp. 248). São Paulo: Casa do Psicólogo. 
Wechsler, S. (2012). O desenho da figura humana: medida cognitiva, criativa ou emocional. Em S. M. Wechsler \& T. C. Nakano. O desenho infantil: forma de expressão cognitiva, criativa e emocional. (Ed.), (pp. 33-65). São Paulo: Casa do Psicólogo.

Zanon, C., Bandeira, C. M., \& Giacomoni, C. H (no prelo). Versão Reduzida da Escala de Satisfação de Vida para Crianças.

\section{Sobre as autoras}

Vanisa Fante Viapiana é psicóloga, especialista em avaliação psicológica pela UFRGS e mestranda em Psicologia com Ênfase em Cognição Humana pela PUCRS.

Cláudia de Moraes Bandeira é doutora e mestre em Psicologia do Desenvolvimento pela UFRGS. Graduada em Psicologia pela Pontifícia Universidade Católica do Rio Grande do Sul e especialista em Psicoterapia de Abordagens Sistêmicas pela Prontamente Clínica da Família. Professora no UNILASALLE.

Claudia Hofheinz Giacomoni é psicóloga e doutora em Psicologia pela UFRGS, professora do Departamento de Psicologia do Desenvolvimento e da Personalidade e do Programa de Pós-Graduação em Psicologia do Instituto de Psicologia da Universidade Federal do Rio Grande do Sul e pesquisadora do Laboratório de Mensuração da UFRGS e coordenadora do Núcleo de Psicologia Positiva. 